\section{THE DOPPLER VELOCIMETRY IN TWIN PREGNANCY COMPLICATED BY INTRAUTERINE GROWTH RESTRICTION IS PREDICTIVE OF NEONATAL OUTCOME?}

S. Visentin ${ }^{1,2}$, S. Vedovato ${ }^{3}$, C. Stoppani ${ }^{1,2}$, F. Cavallin ${ }^{2}$, D. Trevisanuto ${ }^{3}$, V. Zanardo ${ }^{3}$, E. Cosmi ${ }^{2}$, Study Group of Maternal fetal medicine of University of Padua

${ }^{1}$ Padua University, ${ }^{2}$ Department of Gynecological Science and Human Reproduction, ${ }^{3}$ Department of Pediatrics, Padua University, Padua, Italy

Objective: to assess whether Doppler velocimetry is predictive of neonatal outcome in bichorionic and monochorionic twin pregnancies complicated by intrauterine growth restriction.

Methods: were collected retrospectively data of all twin pregnancies complicated by intrauterine growth restriction during the period between January 2006 and December 2009.

Intrauterine Growth Restricted fetuses (IUGR) were defined as fetuses with estimated fetal weight $(E F W)<10^{\text {th }}$ percentile with abnormal Doppler velocimetry (umbilical artery $\mathrm{PI}>2 \mathrm{SD}$ ) and Small for Gestational Age (SGA) fetuses with EFW< $10^{\text {th }}$ percentile and normal Doppler velocimetry. SGA infants were defined birth weight below the 10th percentile according to reference curves for gestational age. Were registered recovery in NICU and neonatal outcomes.

Results: among 349 infants considered, the incidence of selective intrauterine growth restriction with alteration of Doppler velocimetry was found to be $5.7 \%$, while of SGA of $20.3 \%$. The condition of IUGR was associated with an inferior birth weight (median 1241, IQR 785-1700 $p<0.0001$ ) and gestational age at delivery (median 31 gestational age, IQR 30-33 $\mathrm{p}<0.0001)$. The Apgar at $5^{\prime}$ is also lower than the non IUGR group ( 8 vs 9 , range $8-9 p=0.03$ ). There was no statistically significant differences between IUGR and non IUGR group; moreover there wasn't a significant different outcome between IUGR and SGA neonates.

Conclusions: alterations in Doppler velocimetry allow to identify a group of fetuses with intrauterine growth restriction at risk. In terms of neonatal outcome there are no significant differences of morbidity and mortality between IUGR and no IUGR.

\section{PROCOLLAGEN TYPE III PROPEPTIDE LEVELS AMONG PRETERM INFANTS IN NEONATAL INTENSIVE CARE UNIT}

\author{
R.N. Said ${ }^{1}$, A.A.E.A. Fattouh², R.M. Bar El Din', \\ M.T. Saleh ${ }^{2}$, A.S. El Wakkad ${ }^{3}$ \\ ${ }^{1}$ Pediatrics, Faculty of Medicine Cairo University, \\ ${ }^{2}$ Pediatrics, ${ }^{3}$ Clinical Pathology, National Research \\ Center, Cairo, Egypt
}

Aim: To determine levels of the procollagen III propeptide (PIIINP) among preterm neonates whether septic, mechanically ventilated or growers .Also, to evaluate the clinical usefulness of the marker in early detection of fibrosis associated with mentioned neonatal disorders and to correlate it with the ventilation duration and other sepsis markers.

Patients and Methods: We prospectively studied 55 neonates born at Kasr Al Aini obstetric hospital. Within 48 hours of age, 35 preterm infants with gestational age $\leq 35$ weeks were stratified into 3 groups: Group (1) Septic preterms ( $n=15)$; Group (2) Mechanically ventilated $(n=11)$ and Group (3) Growers: Preterms whose weight< 1600 gms admitted for weight gain $(n=9)$. Twenty healthy full term infants were enrolled as control group. Procollagen III propeptide (PIIINP) was measured by radioimmunoassay.

Results: Serum PIIINP is significantly higher in cases than controls. The highest levels were in the ventilated group, followed by the septic one and lastly the Grower group. Serum (PIIINP) levels were significantly higher in males than females (in cases and controls ) and in those who developed bronchopulmonary dysplasia (BPD) Serum procollagen III is significantly positively correlated with ventilation duration and negatively correlated with gestational age and anthropometric measurements.

Conclusion: Serum PIIINP levels are higher in septic, ventilated especially those complicated with BPD and growing preterm which is implicated in the pathogenesis of these disorders.Further research is needed to evaluate the clinical usefulness of the serum PIIINP as a potential marker for neonatal sepsis and BPD. 